\title{
Investigation of pump-ejector systems characteristics for water alternating gas injection
}

\author{
A.N. Drozdov \\ Doctor of Engineering Sciences, Professor, Peoples Friendship University of Russia, Moscow, Russia \\ S.D. Karabaev \\ Post-graduate student of Peoples Friendship University of Russia, Moscow, Russia \\ N.P. Olmaskhanov \\ Post-graduate student of Peoples Friendship University of Russia, Moscow, Russia \\ Y.A. Gorbyleva \\ Post-graduate student of Peoples Friendship University of Russia, Moscow, Russia \\ I.M. Narozhnyy \\ Senior lecturer of Peoples Friendship University of Russia, Moscow, Russia \\ E.I. Gorelkina \\ Post-graduate student of Peoples Friendship University of Russia, Moscow, Russia
}

\begin{abstract}
In this article, the experiments conducted to increase the efficiency of the use of pumping-ejector systems used for the enhanced oil recovery metod. This method is the water alternating gas injection. Water alternating gas injection implies the injection of alternately water and gas, or a water-gas mixture - simultaneous water alternating gas. The purpose of these experiments is to study the jet apparatus characteristics at high pressures at the ejector's intake $(0-0.3 \mathrm{MPa})$, as well as to develop a new calculation methodology for the rheometer. Studies were conducted on a laboratory bench, allowing research at various gas flow rates. The article presents new dependencies for the three possible stand designs that allow recalculation of gas flow. The new rheometer calculation methodology can reduce the inaccuracy from $20 \%$ to $1.8 \%$. The results of experiments that, the maximum value of the ejector efficiency is equal to $32.5 \%$, and the injection rate varies from $1-1.14$, under these experiments conditions.
\end{abstract}

Keywords: pump-ejector systems, characteristic of liquid-gas ejector, water alternating gas injection

\section{INTRODUCTION}

Simultaneous water alternating gas (SWAG) injection is a combination of conventional waterflooding and gas injection techniques. Unlike water, which takes up small hydrophilic pores and bottleneckings in the flooded zone of the reservoir, pumped gas as a non-wetting phase takes large hydrophobic pores, and the top of the reservoir under the action of gravitational forces (Drozdov et al. 2006). These aspects of oil displacement have led to reasonability of maintaining reservoir pressure by water and gas injection. At the same time, the displacement profile is also aligned, and the formation coverage is increased. Various authors carried out laboratory filtration experiments on the displacement of oil and hydrocarbon liquids from the reservoir by water, gas and various combinations of these agents. The results of experiments (Piyakov et al. 1992) on the use of water alternating gas (WAG) injection showed that the 
injection of gas and water, regardless of the type of gas used and the stage of flooding, contributes to an increase in the displacement efficiency. The value of the increase of displacement efficiency is a function of the gas agent composition. The minimum increase of $7-8 \%$ was obtained in experiments using nitrogen; upon injection of dry hydrocarbon gas, the increase was $15-16 \%$; with an increase in the content of intermediate components $\mathrm{C}_{2}-\mathrm{C}_{4}$ in a hydrocarbon gas, the displacement efficiency increased. The series of experiments on core samples (Fatemi et al. 2013), taking into account the wettability of the reservoirs, proves the effectiveness of the use of WAG compared to injecting water or gas separately.

Experiments (Stepanova 2003) suggested that the most significant effect of WAG was observed for media with mixed wettability, when hydrophobic surfaces form through paths along large pores, while smaller ones remain hydrophilic. The direct observation of physical processes occurring in a porous medium during WAG was carried out on the water-wet glass micromodel (Sohrabi et al. 2000). Various water alternating gas technologies over the past decades have been applied at almost a hundred fields in the world, both abroad and in our country. The successful ratio of using WAG according to data in foreign practice exceeds $90 \%$ (Berge 2002).

With the simultaneous injection of water and gas, the greatest effect according to the data (Egorov 2006, Liskevich 1973, Drozdov 2006) is achieved when the gas content of the watergas mixture is in the range of $25-75 \%$. Now abroad, dry or enriched hydrocarbon gases, as well as carbon dioxide, are usually injected into WAG technologies. The most commonly reported increase in oil recovery during WAG is approximately $5 \%$, but, as noted in some fields, oil recovery may increase by $20 \%$. Studies (Lei et al. 2016) have shown the efficiency of WAG $\mathrm{CO}_{2}$ for multilayer formation.

In the article (Christensen et al. 2001) extensive review of the WAG injection process has been presented. The increase in oil production of the considered fields is about $5-10 \%$. To obtain the effect of WAG and to avoid early breakthrough of the injection gas, it is necessary to have a good idea of the phase of the behavior of the reservoir oil and injection gas mixtures, as well as the heterogeneity of the reservoir.

The experimental study of WAG and SWAG injection schemes with $\mathrm{CO}_{2}$ (Kamali 2017) is aimed at determining the function of co-optimization function for $\mathrm{CO}_{2}$ storage and incremental oil production. Experiments are run to represent immiscible, near-miscible, and miscible displacements. Numerical simulations show a remarkable reduction in gas relative permeability for the WAG and SWAG displacements compared with continuous-gas-injection displacements, as a result of which the vertical-sweep efficiency of $\mathrm{CO}_{2}$ is improved.

A comprehensive review of WAG field displacements and their effect on oil recovery and produced-gas/water ratio has been presented in the literature (Stalkup 1983; Christensen et al. 2001).

The vast simulation study (Nygård \& Andersen 2020) has been performed investigating immiscible WAG injection focusing on mechanisms such as mobility, gravity, injected volume fractions, reservoir heterogeneity, gas entrapment, and relative permeability hysteresis.

Scientists (Hoare \& Coll 2018, Kumar et al. 2017, Khorsandi et al. 2017; Alzayer \& Sohrabi 2018) note the influence of hysteresis relative permeability on the effectiveness of WAG. It is often expected that the gas phase will show large hysteresis effects (Hoseini et al. 2011). Hysteresis lowers gas mobility and hence improves the gas/oil mobility ratio and reduces gravity segregation. The first effect is always positive, but the second is mainly positive in more uniform reservoirs where gravity segregation has a negative effect on recovery. In heterogeneous reservoirs, reducing gravity segregation can lead to the oil in low-permeability layers remaining unswept. (Nygård \& Andersen 2020)

Most foreign fields with WAG technologies are located in Canada, the USA and Norway (Skauge \& Stensen 2003, Christensen et al. 2001, Cone 1970, Quale et al. 2000, Righi \& Pascual 2007, Shi et al. 2008, Stoisits et al. 1995, Choudhary et al. 2011, Gbadamosi et al. 2018, Olalotiti-Lawal et al.2018).

In Russia, experimental work on the WAG was carried out at the Sovetskoye and Vakhskoye (Kryuchkov 2002), Ilishevskoye (Shuvalov et al. 2008), Alekseevskoye (Vafin 2008, Zaripov 2006), Novogodnee (Zakirov et al. 2006), Vostochno-Perevalnoye (Zatsepin \& Chernikov 2007, Chubanov et al. 2008) and at the Sredne-Hulymskoye fields (Chubanov et al. 2008). 
In foreign practice, complex and expensive equipment that requires large capital investments, high-quality maintenance and competent operation - gas treatment units, pumps and high pressure compressors - is used to implement gas and water exposure. Attempts to apply the WAG compressor technology at the Samotlorskoye and Novogodnoye fields under Russian conditions, despite the enormous costs, were ultimately discontinued.

So the traditional technology of SWAG injection is not widely used in domestic oil fields. The main problem is the absence of effective processes and technologies for water and gas pumping into injection wells. In this situation, the use of jet technology, in particular pumpejector systems, may turn out to be a very attractive solution.

In previously published papers (Krasilnikov 2010, Drozdov et al. 2008), laboratory studies of pump-ejector systems were carried out at constant pressure at the jet apparatus intake $\mathrm{P}_{\text {in }}$ $(0 ; 0.5 \mathrm{MPa})$ with different values of relative power pressure drop created in the ejectors working nozzle $\Delta \mathrm{P}_{\mathrm{p}}$. In this article, the characteristics of an ejector with a constant $\Delta \mathrm{P}_{\mathrm{p}}$ with a relative outlet pressure drop $\Delta \mathrm{P}_{\mathrm{o}}$ created by a jet apparatus were considered.

\section{PURPOSE}

The purpose of this work is to characterize the pump-ejector system at high pressures at the jet apparatus intake within $0-0.3 \mathrm{MPa}$. Additionally, the differential pressure gauge should be calibrated with the creation of a new calculation methodology.

\section{MATERIALS AND METHODS}

Investigations of the pump-ejector system was carried out on the laboratory bench (Figure 1) which worked in the following way. The power liquid (water) from the tank 1 enters the intake of a multistage centrifugal pump 2. Next, the power fluid is forced to the nozzle of the jet apparatus 3 and injects, depending on the experimental conditions, either gas from tank 1 or gas from the atmosphere, which is regulated by valves 12 and 13 .

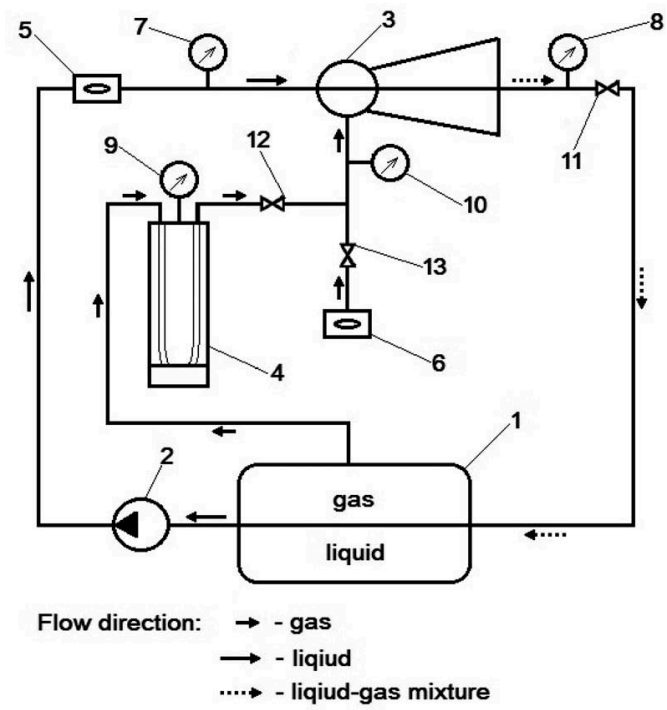

Figure 1. Diagram of the stand for the pump-ejector system investigation: 1 - liquid-gas tank; 2 - multistage centrifugal pump; 3 - ejector; 4 - differential pressure gauge; 5 - fluid meter; 6 - gas meter; 7, 8, 9 manometers; 10 - pressure-vacuum meter; 11, 12, 13 - control valves and gate valves. 
The flow rate of the power fluid injected into the nozzle is measured by a fluid meter 5 . Depending on the conditions, the flow rate of the injected gas is measured by a differential pressure gauge 4 when high pressure gas is injected, or by a gas meter 6 when atmospheric air is injected. The pressures in front of the working nozzle of the jet apparatus and at the output from its diffuser were measured using manometers 7 and 8 . The pressure at the ejector's intake was measured with a pressure - vacuum meter 10. Different operating modes and pressures were generated by a control valve 11 .

Measured parameters during the experiment: fluid rate, gas rate from the tank, gas rate from the atmosphere, power pressure in front of the nozzle, pressure at the ejector's intake and output pressure. The efficiency of the ejector $\eta_{g}$ during gas injection is determined by the ratio of the effective power $N_{e}$ to total power $N_{t}$ (Drozdov 2014):

$$
\begin{aligned}
& \eta_{g}=\frac{N_{e}}{N_{t}}=\frac{\mathrm{Q}_{g . i n} P_{i n} \ln \frac{\mathrm{P}_{\mathrm{o}}}{\mathrm{P}_{\text {in }}}}{\mathrm{Q}_{p}\left(P_{o}\right)} \\
& =\frac{\mathrm{u}_{\mathrm{g}} p_{\text {in }} \ln \frac{\mathrm{P}_{\mathrm{o}}}{\mathrm{P}_{\text {in }}}}{\left(P_{p}-P_{0}\right)}
\end{aligned}
$$

where $\mathrm{Q}_{\mathrm{g} . \mathrm{in}}$ - volumetric gas rate in the suction chamber;

$p_{\text {in }}$ - pressure in the suction chamber (intake chamber);

$\mathrm{P}_{\mathrm{o}}$ - outlet pressure of the ejector;

$P_{p}$ - power pressure in front of the nozzle;

$\mathrm{Q}_{p}$ - volumetric power fluid rate;

$\mathrm{u}_{\mathrm{g}}=\mathrm{Q}_{\mathrm{g} \text {.in }} / \mathrm{Q}_{p}$ is the volumetric injection coefficient in the suction chamber.

Technical water is used as a power liquid and air as a gas. Relative pressure drop:

$$
\frac{\Delta \mathrm{P}_{\mathrm{c}}}{\Delta \mathrm{P}_{\mathrm{p}}}=\frac{P_{c}-P_{\pi \mathrm{p}}}{P_{p}-P_{\pi \mathrm{p}}}
$$

where $\Delta \mathrm{P}_{\mathrm{c}}, \Delta \mathrm{P}_{\mathrm{p}}$ - pressure drops created by the jet apparatus and the working nozzle of the ejector respectively.

Differential pressure gauge DT-50 was used to determine the gas rate from the tank. Generally, it is used to measure the flow rate of non-aggressive gases, vapors and liquids by determining the pressure drop across the orifice plate or other throttle body.

Additional tubes were used for the accuracy and to increase the measurement range for gas rate (Figure 2). Were used 3 schemes of gas flow which dependences on the degree of gas rate.

The "right" scheme is used at pressures $\mathrm{P}<0.18 \mathrm{MPa}$, which is activated by opening the valve 7 and closing the valve 6 . The "left" scheme is used at pressures of $0.18<\mathrm{P}<0.26 \mathrm{MPa}$, where the valves open vice versa to the "right" scheme. The "general" scheme is used when $\mathrm{P}>0.26 \mathrm{MPa}$, where both valves open. Theoretically, after getting the calibration curve at any pressure level, other pressure values can be obtained using the following equation:

$$
Q_{2}=Q_{1} \sqrt{\frac{\lambda_{1}}{\lambda_{2}} \frac{P_{1}}{P_{2}}}
$$

where $Q_{1}, Q_{2}$ - the gas rates in the rheometer at pressures $P_{1}$ and $P_{2}$ respectively; $\lambda_{1}, \lambda_{2}-$ resistance values at $P_{1}$ and $P_{2}$ respectively.

For a quadratic zone of gas flow in a tube, as is well known $\lambda_{1}=\lambda_{2}$, where at the same water level difference in the differential pressure gauge, we obtain: 


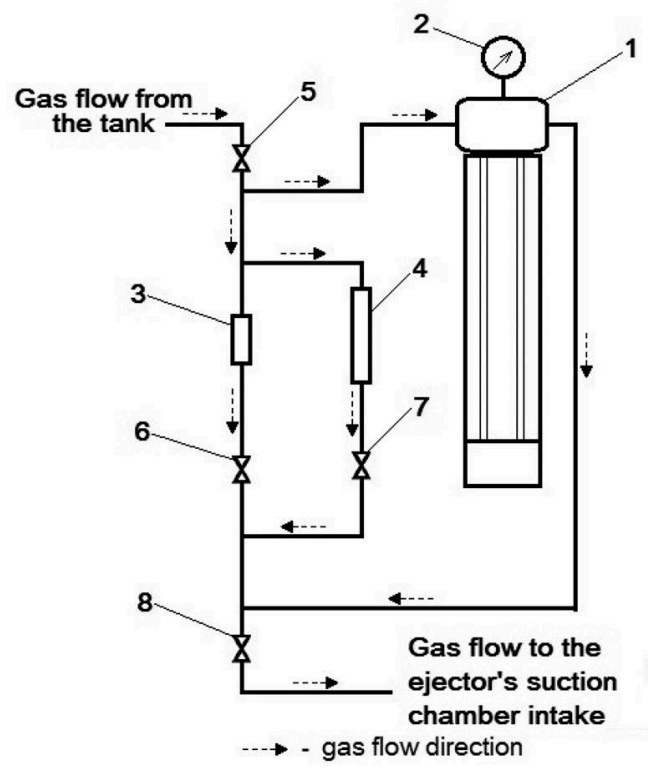

Figure 2. Diagram of a differential pressure gauge with variable parameters of the measurement range: 1 - differential pressure gauge; 2 - manometer; 3 - copper tube (shortened); 4 - copper tube (elongated); 5, $6,7,8$ - valves and gate valves.

$$
Q_{2}=Q_{1} \sqrt{\frac{P_{1}}{P_{2}}}
$$

In article (Drozdov 1982) it is pointed out that in the calibration process where brass tubes with an internal diameter of 4-6 $\mathrm{mm}$ was used at pressure under 0.6 MPa, the resistance values are not equal $\lambda_{1} \neq \lambda_{2}$. Analytical determination of $\lambda$ with the required accuracy depending on pressure and flow rate by formula (3) is difficult to perform. Due to it, the calibration dependencies were built at the same pressures as in the experiments pressures values. Since our studies are carried out with $6 \mathrm{~mm}$ tubes at pressures below $0.6 \mathrm{MPa}$, it is necessary to introduce correction factors for formula (2).

Calibration of the differential pressure gauge was carried out in this way. Calibration curves were registered at pressures of $0.1 ; 0.14 ; 0.18 ; 0.22 ; 0.26 ; 0.3 \mathrm{MPa}$. Gas rates at appropriate pressures were calculated based on $0.1 \mathrm{MPa}$ by the formula (4). After that, the deviations between the calculated and actual values were estimated. According to the deviations, correction factors were found, and flow rates based on new formula were calculated. Next will be presented the results.

\section{RESULTS AND DISCUSSION}

This section presents the results of experimental studies of the pump-ejector system and calibration of the differential pressure gauge.

\subsection{Differential pressure gauge}

Because of the calibration carried out, it was possible to achieve sufficient accuracy of the new calculation methodology (Table 1, Table 2, Table 3). The following formula was obtained for the "right" scheme: 
Table 1. The results of the calibration of the differential pressure gauge for the "right" scheme.

\begin{tabular}{lll}
\hline $\mathrm{P}, \mathrm{MPa}$ & Old method $(\Delta, \%)$ & New method $(\Delta, \%)$ \\
\hline 0,14 & 9,9 & 1,14 \\
0,18 & 16,8 & 1,78 \\
0,22 & 22,7 & 1,71 \\
0,26 & 26,3 & 1,62 \\
0,3 & 28,7 & 1,35 \\
\hline
\end{tabular}

Table 2. The results of the calibration of the differential pressure gauge on the "left" scheme.

\begin{tabular}{lll}
\hline $\mathrm{P}, \mathrm{MPa}$ & Old method $(\Delta, \%)$ & New method $(\Delta, \%)$ \\
\hline 0,14 & 9,1 & 1,37 \\
0,18 & 15,4 & 1,11 \\
0,22 & 18,9 & 0,99 \\
0,26 & 23,1 & 1,01 \\
0,3 & 25,4 & 1,15 \\
\hline
\end{tabular}

Table 3. The results of the calibration of the differential pressure gauge on the "common" scheme.

\begin{tabular}{lll}
\hline $\mathrm{P}, \mathrm{MPa}$ & Old method $(\Delta, \%)$ & New method $(\Delta, \%)$ \\
\hline 0,14 & 9,7 & 1,86 \\
0,18 & 14,7 & 1,5 \\
0,22 & 20,0 & 1,66 \\
0,26 & 23,0 & 1,83 \\
0,3 & 25,2 & 1,44 \\
\hline
\end{tabular}

$$
\mathrm{Q}_{2}=\mathrm{Q}_{1} * \sqrt{\frac{\mathrm{P}_{1}}{\mathrm{P}_{2}}} *\left(-5,04 *(\Delta P)^{2}+2,38 *(\Delta P)+1,01\right)
$$

where $\mathrm{Q}_{1}, \mathrm{Q}_{2}$ - volumetric gas rates in differential pressure gauge at pressure $\mathrm{P}_{1}, \mathrm{P}_{2}$ respectively;

$$
\Delta P=P_{2}-P_{1}
$$

During the calibration of the differential pressure gauge on the "right" scheme, the following results are obtained:

- according to the old method, the averaged inaccuracy is $20.88 \%$;

- according to the new method, the averaged inaccuracy does not exceed $1.8 \%$.

The next formula was obtained for "left" scheme: 


$$
\mathrm{Q}_{2}=\mathrm{Q}_{1} \times \sqrt{\frac{\mathrm{P}_{1}}{\mathrm{P}_{2}} \times\left(-3,08 *(\Delta P)^{2}+1,75 *(\Delta P)+1,03\right)},
$$

During the calibration of the differential pressure gauge on the "right" scheme, received the following indicators:

- according to the old method, the averaged inaccuracy is $18.52 \%$;

- according to the new method, the averaged inaccuracy does not exceed $1.9 \%$.

The next formula was obtained for "common" scheme:

$$
\mathrm{Q}_{2}=\mathrm{Q}_{1} \times \sqrt{\frac{\mathrm{P}_{1}}{\mathrm{P}_{2}} \times\left(-3,53 *(\Delta P)^{2}+1,83 *(\Delta P)+1,03\right),}
$$

During the calibrating for the "common" system, we obtained the following indicators:

- according to the old method, the averaged inaccuracy is $18.52 \%$

- according to the new method inaccuracy does not exceed $1.9 \%$.

\subsection{Characteristics of the pump-ejector system}

After the calibration of the differential pressure gauge, the pressure-energy characteristics of the ejector were obtained (Figure 3, Figure 5). The maximum efficiency of ejector $\eta_{g}$ during gas injection is obtained 0.325 , where the relative pressure drop is $\Delta \mathrm{P}_{\mathrm{o}} / \Delta \mathrm{P}_{\mathrm{p}}=0.315$, injection coefficient $\mathrm{u}_{\mathrm{g}}$ in the suction chamber is 1.13 at intake pressure $\mathrm{P}_{\text {in }}=0.26 \mathrm{MPa}$ and outlet pressure $\mathrm{P}_{\mathrm{o}}=0.636 \mathrm{MPa}$. The minimum efficiency of ejector $\eta_{g}$ during gas injection is obtained 0.245 , where the relative pressure drop is $\Delta \mathrm{P}_{\mathrm{o}} / \Delta \mathrm{P}_{\mathrm{p}}=0.294$, injection coefficient in the suction chamber is $u_{g}=1.14$ under intake pressure $P_{\text {in }}=0.05 \mathrm{MPa}$ and outlet pressure $\mathrm{P}_{\mathrm{o}}=0.397$ $\mathrm{MPa}$. It can also be noted that in the pressure range $\mathrm{P}_{\text {in }}=0.05-0.3 \mathrm{MPa}$ the injection coefficient in the suction chamber is varies from 1 to 1.14 .

After that, a graph of maximum efficiencies $\eta_{g}$ was plotted to the function of intake pressures $\mathrm{P}_{\text {in }}$ (Figure 4.). This graph shows that the difference between the maximum and

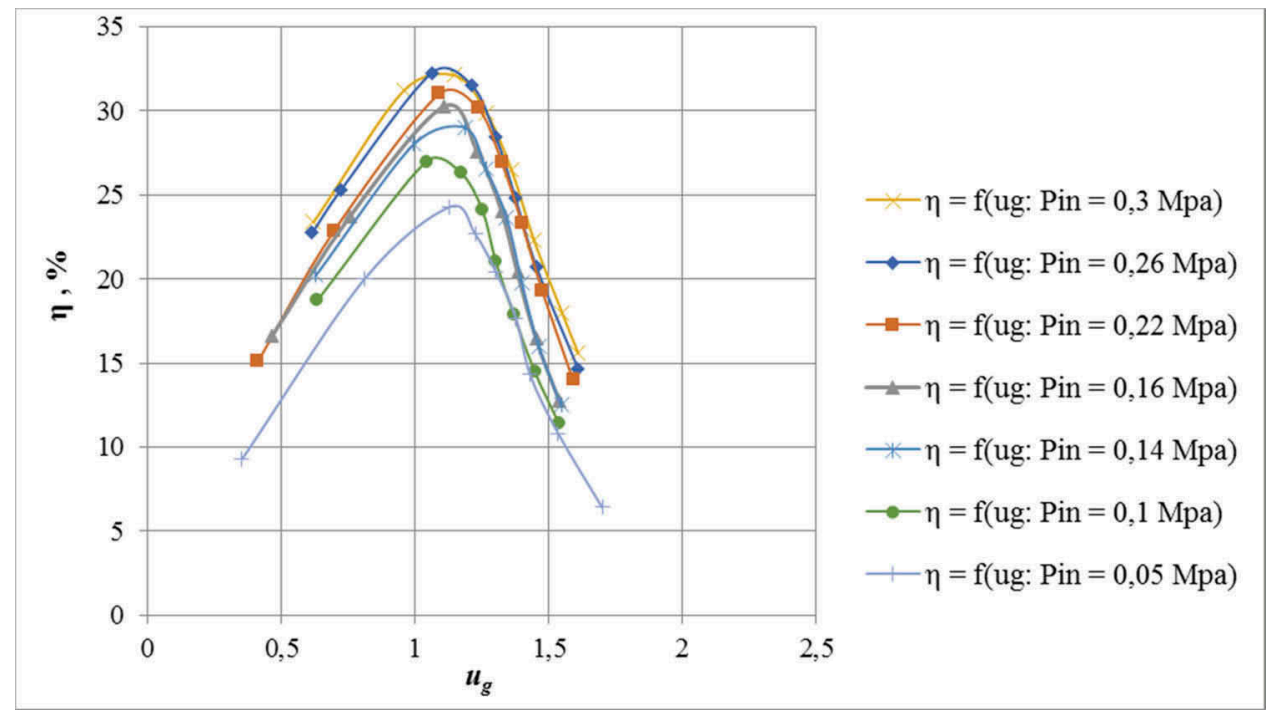

Figure 3. The ejector efficiencies $\eta_{g}$ to the function of injection coefficient $u_{g}$. 


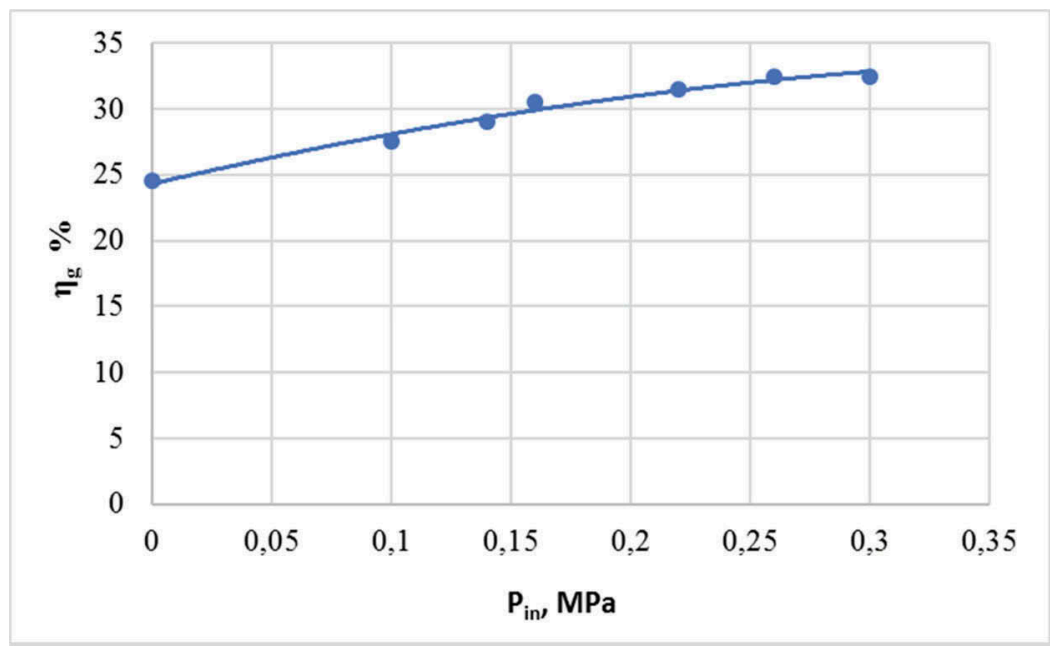

Figure 4. The maximum efficiencies $\eta_{g}$ to the function of intake pressure $\mathrm{P}_{\mathrm{in}}$.

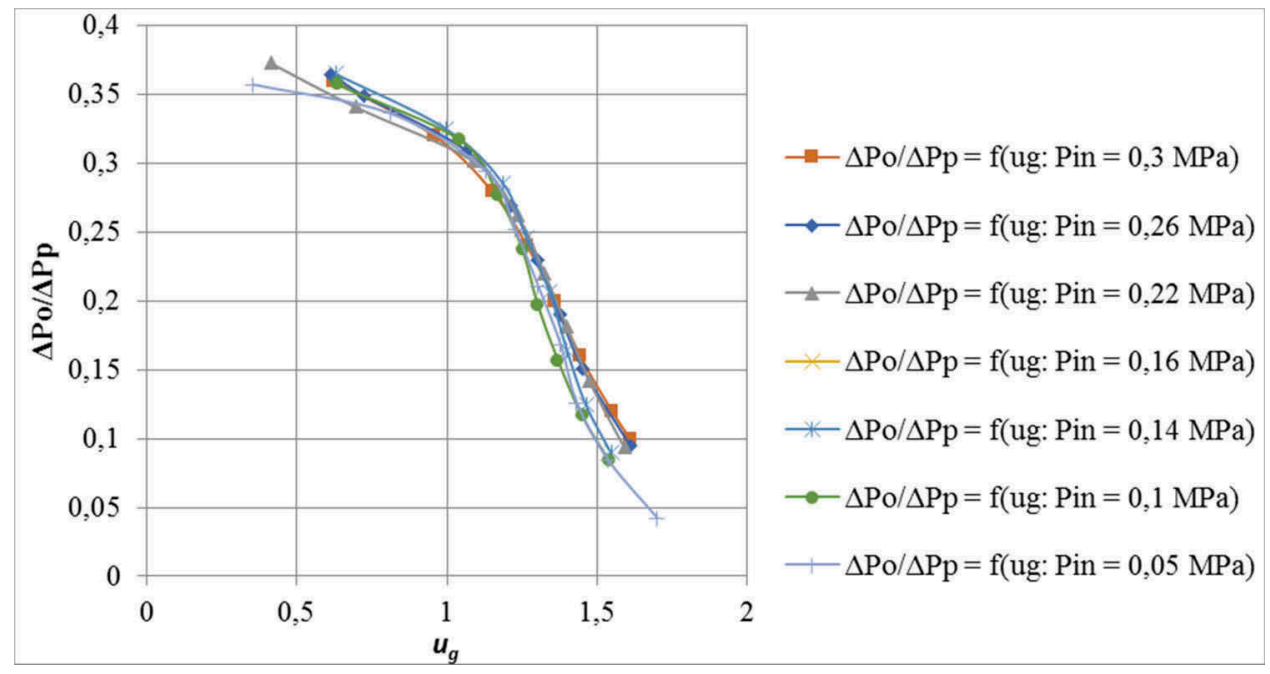

Figure 5. The relative pressure drop $\Delta \mathrm{P}_{\mathrm{o}} / \Delta \mathrm{P}_{\mathrm{p}}$ to the function of injection coefficient $u_{g}$.

minimum values is 0.078 . The value of $\eta_{g}$ gradually increases to $\mathrm{P}_{\text {in }}=0.26 \mathrm{MPa}$ and after this point some decline is observed.

\section{CONCLUSION}

Research and calculations have shown that, the new calculation methodology of the differential pressure gauge makes it possible to calculate gas rates with an inaccuracy of no more than $1.8 \%$.

The obtained pressure-energy characteristics for high pressures at the ejector's intake in the range from 0.05 to $0.3 \mathrm{MPa}$ allow us to conclude that the maximum efficiency at this condition, do not exceed $32.5 \%$. The volumetric injection coefficient rates range from 1 to 1.14 . It is 
worth noting that after the intake pressure of $0.26 \mathrm{MPa}$, there is some decrease in the efficiency, which can be assessed in further studies at higher intake pressure $\mathrm{P}_{\text {in }}$.

\section{ACKNOWLEDGMENT}

The publication has been prepared with the support of the «RUDN University Program 5-100».

\section{REFERENCES}

Alzayer, H. \& Sohrabi, M. 2018. Water-Alternating-Gas Injection Simulation-Best Practices SPE EOR Conference at Oil and Gas West Asia, 26-28 March: SPE-190346-MS Oman: Muscat

Berge, L.I., Stensen, J.A., Crapez, B. \& Quale, E.A. 2002. SWAG Injectivity Behavior Based on Siri Field DataImproved Oil Recovery Symposium, 13-17 April: SPE 75126 Oklahoma: Tulsa.

Choudhary, M.K., Dezabala, E., Solis, H.A. \& Narvaez, J. Z. Z. 2011. Design, Implementation and Performance of a Down-Dip WAG Pilot International Petroleum Technology Conference, 15-17 November: IPTC-14571-MS Thailand: Bangkok

Christensen, J. R., Stenby, E. H. and Skauge, A. 2001. Review of WAG Field Experience. SPE Res Eval \& Eng 4 (2): 97-106.

Chubanov, O.V., Harlanov, S.A. \& Nurgaliev, R.G. 2008. Development and implementation of water alternating gas injection methods for enhanced oil recovery at RITEK Territory of NEFTEGAZ vol. 9: $42-48$

Cone, C. 1970. Case history of the University Block-9 (Wolfcamp) field - a gas-water injection secondary recovery project Journal of Petroleum Technology XII, vol. 22:1485-1491.

Drozdov, A.N. 1982. Development of methods for calculating the characteristics of a submersible centrifugal pump for the operation of wells with low pressures at the entrance to the pump Cand. Sci. Dissertation Moscow p 212

Drozdov, A.N., Egorov, Yu.A., Verbitsky, V.S., Dengaev, A.V. \& Lambin, D.N. 2006. Methods and technology of water alternating gas injection influence to oil reservoirs Territory NEFTEGAZ vol 2: 54-58

Drozdov, A.N., Krasilnikov, I.A. \& Verbitsky V.S. 2008. Investigation of the characteristics of pumping-ejector systems for water-gas impact on the formation Territory NEFTEGAZ vol 2: 60-63

Drozdov, A.N. 2014. Problems of simultaneous water alternating gas injection and its solutions Oil industry vol 2: 2-6

Egorov, Yu.A. 2006 Development of water-alternated-gas technology using pump-ejector systems to increase oil recovery Cand. Sci. Dissertation Moscow p 169

Fatemi, S.M. \& Sohrabi, M. 2013. Experimental investigation of near-miscible water-alternating-gas injection performance in water-wet and mixed-wet systems SPE Journal vol. 18(1): 114-123.

Gbadamosi, A. O., Kiwalabye, J., Junin, R. \& Augustine, A. 2018. A review of gas enhanced oil recovery schemes used in the North Sea Journal of Petroleum Exploration and Production Technology vol. 8:1373-1387

Hoare, G. \& Coll, C. 2018. Effect of Small/Medium Scale Reservoir Heterogeneity on the Effectiveness of Water, Gas and Water Alternating Gas WAG Injection. SPE Europec featured at the 80th EAGE Conference and Exhibition, 11-14 June: SPE-190855-MS Denmark: Copenhagen

Hoseini, J., Masoudi, R., Mousavi Mirkalaei, S. M. et al. 2011. Investigating the Effect of Hysteresis Modelling on Numerical Simulation of Immiscible WAG Injection. International Petroleum Technology Conference, 15-17 November: IPTC-15055-MS. Thailand: Bangkok

Kamali, F., Hussain, F. \& Cinar, Y. 2017. An Experimental and Numerical Analysis of Water-Alternating-Gas and Simultaneous-Water-and-Gas Displacements for Carbon Dioxide Enhanced Oil Recovery and Storage SPE Journal vol. 22 (2): 18

Khorsandi, S., Li, L., \& Johns, R. T. 2017. Equation of State for Relative Permeability, Including Hysteresis and Wettability Alteration SPE Journal vol. 22 (6):1915-1928.

Krasilnikov, I.A. 2010. Development of methods for calculating the characteristics of liquid-gas ejectors for well operation and water alternating gas influence on the reservoir using pump-ejector systems Cand. Sci. Dissertation Moscow p 144

Kryuchkov, V.I. 2002. The use of water-gas systems based on petroleum gas to increase oil recovery. Cand. Sci. Dissertation Bugulma p 193 
Kumar, J., Agrawal, P. \& Draoui, E. 2017. A Case Study on Miscible and Immiscible Gas-Injection Pilots in a Middle East Carbonate Reservoir in an Offshore Environment. SPE Res Eval \& Eng 20 (1): 19-29. SPE-181758-PA.

Lei, H., Yang, S., Zu, L., Wang, Z. \& Li, Y. 2016. Oil recovery performance and $\mathrm{CO}_{2}$ storage potential of $\mathrm{CO}_{2}$ water-alternating-gas injection after continuous $\mathrm{CO}_{2}$ injection in a multilayer formation Energy \& fuels vol. 30 (11): 8922-8931.

Liskevich, E.I. \& Ostrovsky, Yu.M. 1973. Oil displacement by gas-water mixtures Oil field development, proceedings of UkrNIIIPND vol. 11-12: 233-240.

Nygård, J. I. \& Andersen, P. Ø. 2020. Simulation of Immiscible Water-Alternating-Gas Injection in a Stratified Reservoir: Performance Characterization Using a New Dimensionless Number SPE Journal SPE-200479-PA

Olalotiti-Lawal, F., Onishi, T., Datta-Gupta, A., Fujita, Y., Watanabe, D. \& Hagiwara, K. 2018. PostCombustion $\mathrm{CO}_{2}$ WAG Pilot in a Mature Field: Model Calibration and Optimization SPE Annual Technical Conference and Exhibition, 24-26 September: SPE-191472 Texas: Dallas

Piyakov, G.N., Yakovlev, A.P., Kudashev, R.I. \& Romanova, E.I. 1992. The study of the efficiency of water alternating gas injection (on the example of the $\mathrm{Yu}_{1}$ layer of the Kogalymskoye field) Oil industry vol. 1: 38-39.

Quale, E.A., Crapez, B., Stensen, J.A. \& Berge, L.I.B. 2000. SWAG Injection on the Siri Field - An Optimized Injection System for Less Cost, SPE 65165, SPE European Petroleum Conference held in Paris, France, October, 24-25 France: Paris

Righi, E.F. \& Pascual, M. 2007. Water-Alternating-Gas Pilot in the Largest Oil Field in Argentina: Chihuido de la Sierra Negra, Neuquen Basin. SPE Latin American and Caribbean Petroleum Engineering Conference, 15-18 April 2007: SPE 108031. Argentina: Buenos Aires.

Shi, W., Corwith, J., Bouchard, A., Bone, R. \& Reinbold E. 2008. Kuparuk MWAG Project after 20 Years SPEIDOE Improved Oil Recovery Symposium, 19-23 April 2008: SPE 113933. Oklahoma: Tulsa.

Shuvalov, A.V., Samigullin, I.F. \& Suleymanov, A.A. 2008. Pilot-industrial work at JSOC Bashneft related to the introduction of gas and water alternating gas injection Materials of the VII Interregional Conference "Geology, Minerals, and Geoecology Problems of Bashkortostan, the Urals and Neighboring Territories", 2008, November: 239-241

Skauge, A. \& Stensen, J.O. 2003. A review of field experience in water alternating gas injection. Reports of the 1st International Conference "Oil Recovery - 2003", May 19-23: 119-120. Russia: Moscow

Sohrabi, M., Henderson, G.D., Tehrani, D.H \& Danesh A. 2000. Visualisation of oil recovery by water alternating gas (WAG) injection using high pressure micromodels-water-wet system SPE Annual Technical Conference and Exhibition.

Stalkup, F. I. 1983. Miscible Displacement. Richardson, Texas: Monograph Series, Society of Petroleum Engineers.

Stepanova, G.S. 2003 New methods of gas and water-alternated-gas injection of oil reservoirs Drilling and Oil, vol. 9: 18-20.

Stoisits, R.F., Krist, G.J., Ma, T.D., Rugen, J.A., Kolpak, M.M. \& Payne, R.L. 1995. Simultaneous Water and Gas Injection Pilot at the Kuparuk River Field, Surface Line Impact SPE Annual Technical Conference, Oct. 1995: SPE 30645

Vafin, R.V. 2008. Improving the efficiency of water-alternated-gas technology on the reservoir at the Alekseevskoye field Neftepromyslovoe delo vol. 2: 33-35

Zakirov, S.N., Indrupsky, I.M., Levochkin, V.V., Fakhretdinov, R.N. \& Ostapchuk, S.S. 2006. Wateralternated-gas injection on the Novogodnee field Oil industry, vol.12: 40-43.

Zaripov, M.S. 2006. Improving the technology of water alternating gas injection, preparation and injection of working agents into the reservoir Abstract of Cand. Sci. Dissertation Ufa p 24

Zatsepin, V.V. \& Chernikov, E.V. 2007. Some issues of the implementation of water alternating gas injection at the Vostochno-Perevalnoye field Oil Industry vol. 2: 44-47. 